\title{
An epirubicin-peptide conjugate with anticancer activity is dependent upon the expression level of the surface transferrin receptor
}

\author{
JIADAN YANG ${ }^{1,2}$, QIYU YANG ${ }^{3}, \mathrm{LU} \mathrm{XU}^{2}, \mathrm{JIE} \mathrm{LOU}^{2}$ and ZHI DONG ${ }^{2}$ \\ ${ }^{1}$ Department of Pharmacy, The First Affiliated Hospital of Chongqing Medical University; ${ }^{2}$ Chongqing Key Laboratory \\ of Biochemistry and Molecular Pharmacology, College of Pharmacy, Chongqing Medical University, Chongqing 400016; \\ ${ }^{3}$ Department of Thoracic Oncology, West China School of Medicine, West China Hospital, Sichuan University, \\ Chengdu, Sichuan 610041, P.R. China
}

Received October 26, 2015; Accepted November 2, 2016

DOI: $10.3892 / \mathrm{mmr} .2016 .6004$

\begin{abstract}
Epirubicin (EPI) is one of the most widely used anticarcinogens; however, serious side effects, including cardiomyopathy and congestive heart failure, limit its long-term administration. To overcome this problem, the HAIYPRH peptide ligand was used with EPI in the synthesis of a HAIYPRH-EPI conjugate. The anticancer activity and cellular uptake of the conjugate were measured and evaluated. The results of the present study indicated that the cytotoxicity of HAIYPRH-EPI was correlated with the expression of the cell surface transferrin receptor (TfR). The conjugate exerted high cytotoxicity and proapoptotic function when in an LN229 glioma cell line, which overexpresses surface TfR. It was hypothesized that transferrin (Tf) can promote cytotoxicity. Conversely, the conjugate exhibited very low cytotoxicity and proapoptotic function in a U87 glioma cell line, in which surface TfR expression was undetectable. In addition, fluorescence microscopy and flow cytometry methods were used to evaluate cellular uptake, and the results of these methods were consistent with the present hypotheses. The conjugate cellular uptake of the conjugate in LN229 cells was markedly higher compared with that in U87 cells, and it was hypothesized that Tf can enhance the uptake in LN229 cells. The cytotoxicity of HAIYPRH-EPI was dependent upon the expression of surface TfR. Considering that the majority of cancer cells have high rates of iron uptake and surface TfR is generally overexpressed on cancer cells, it was speculated by the authors that HAIYPRH-EPI may form part of an effective strategy for increasing the selectivity of EPI for cancer cells, as well as
\end{abstract}

Correspondence to: Dr Zhi Dong, Chongqing Key Laboratory of Biochemistry and Molecular Pharmacology, College of Pharmacy, Chongqing Medical University, 1 Youyi Road, Yuanjiagang, Yuzhong, Chongqing 400016, P.R. China

E-mail: dongzhi0326@126.com

Key words: transferrin receptor, peptide, epirubicin, conjugate reducing its systemic toxicity. To confirm the hypothesis, the effects of HAIYPRH-EPI on non-cancerous cell lines were investigated. A future study will examine the side effects of HAIYPRH-EPI, using a suitable delivery system in an animal model.

\section{Introduction}

Epirubicin (EPI) is one of the most well-known and widely used anthracyclines, a class of effective genotoxic anticancer drugs used to treat a variety of human malignancies $(1,2)$. The major mechanism of EPI is inhibition of topoisomerase $2 \alpha$, which induces DNA damage through intercalating the DNA double helix (3). EPI-containing regimens, including 5-fluorouracil/EPI/cyclophosphamide and EPI/cyclophosphamide, are widely used as an adjuvant or neoadjuvant in the treatment of metastatic cancer (3-5). Although these EPI-containing regimens are highly effective, serious side effects, including cardiomyopathy and congestive heart failure, limits their long-term administration (5-7). To circumvent this problem, strategies, including use of prodrugs and targeted delivery systems, have been previously investigated in an attempt to increase the selectivity of EPI for cancer tissues or cells $(6,8,9)$. These strategies require further exploration to identify the most effective method for using EPI, with the fewest side effects.

The transferrin receptor (TfR) is a transmembrane homodimer, able to bind up to two molecules of transferrin (Tf), and internalize them by TfR-mediated endocytosis (10). As a $78 \mathrm{kD}$ a monomeric glycoprotein, Tf is an important chelator primarily involved in serum iron transportation, essential for cellular proliferation (11). TfR is highly expressed on cells that have a high proliferation rate, reflecting the high cellular metabolic requirements for iron (11). Due to their rapid rate of division, the majority of cancer cells have high rates of iron uptake. In addition, TfR is generally overexpressed on cancer cells, $\sim 100$-fold more compared with that on normal cells $(10,12,13)$. Therefore, due to the difference in the expression of TfR between cancerous and normal cells, Tf has been previously used as a targeting ligand $(10,14,15)$. However, it is difficult for $\mathrm{Tf}$ to be synthesized as a conjugate with other 
anticancer drugs, or constructed in a drug targeting delivery system. This is due to its high molecular weight and that it is a competitive inhibitor of endogenous $\mathrm{Tf}$ (10-12).

HAIYPRH, a TfR-targeting peptide, has a high affinity for the TfR with a $K_{\mathrm{d}}$ of $\sim 10 \mathrm{nM}$ (16). In addition, the binding sites on HAIYPRH for the Tf are distinct from the binding sites for the TfR, allowing a co-delivery of HAIYPRH and endogenous Tf to TfR-expressing cells (12). Furthermore, the molecular weight of HAIYPRH is $\sim 1 \mathrm{kDa}$, significantly lower compared with the molecular weight of Tf. These advantageous features of HAIYPRH make it a promising targeting ligand. Oh et al (12) reported that conjugates of HAIYPRH and artemisinin demonstrated significantly increased selectivity for cancer cells.

In the present study, HAIYPRH was used as a targeting ligand to synthesize a HAIYPRH-EPI conjugate. In addition, the anticancer activity and cellular uptake of the conjugate were each evaluated. This study may provide an effective strategy to increase selectivity of EPI for cancer cells, and reduce the systemic toxicity of EPI.

\section{Materials and methods}

Materials. EPI was supplied by Shandong Xinshidai Pharmaceutical Co., Ltd. (Linyi, China). The HAIYPRH-EPI conjugate was synthesized by Hangzhou Dangang Biotechnology Co.,Ltd. (Hangzhou, China). Glutamic acid was used as a linker between HAIYPRH and EPI, and the structure of the conjugate is detailed in Fig. 1. The HAIYPRH-EPI conjugate was identified by liquid chromatography-mass spectrometry. Mass spectrometric detection was performed using a Sciex 2000 QTrap (AB Sciex, Concord, ON, Canada) equipped with electrospray ionization (ESI). The mass spectrometer was employed in the positive ion elctrospray mode with multiple reaction monitoring. The optimal ESI source conditions were as follows: Nebulizer gas pressure, $40.00 \mathrm{psi}$; gas flow rate, $10.00 \mathrm{l} / \mathrm{min}$; ion source temperature, $350^{\circ} \mathrm{C}$; ion transitions of $\mathrm{m} / \mathrm{z}$ 773.8-393.0. Fluorescein isothiocyanate-conjugated anti-human CD71 (anti-TfR) and the isotype fluorescein isothiocyanate-conjugated IgG1 were purchased from eBioscience, Inc. (San Diego, CA, USA). Caspase-3, caspase-8, and caspase-9 activity assay kits were purchased from Beyotime Institute of Biotechnology (Haimen, China). Cell culture reagents and additional reagents were purchased from Gibco (Thermo Fisher Scientific, Inc., Waltham, MA, USA) or Hyclone (GE Healthcare Life Sciences, Logan, UT, USA). All chemicals and reagents were of analytical grade.

Cell lines. U87 and LN229 cells were purchased from Cell Bank of the Type Culture Collection of Chinese Academy of Sciences (Shanghai, China). U87 cells were cultured in Dulbecco's modified Eagle's medium, and LN229 cells were cultured in minimum essential medium. Each type of medium was supplemented with $10 \%$ fetal bovine serum and $1 \%$ penicillin/streptomycin $(100 \mathrm{U} / \mathrm{ml}$ and $100 \mathrm{mg} / \mathrm{ml}$, respectively) and the cells were maintained at $37^{\circ} \mathrm{C}$ in a humidified incubator containing $5 \% \mathrm{CO}_{2}$.

Flow cytometry. Flow cytometric analysis for each glioma cell line was performed, as previously described (17) with

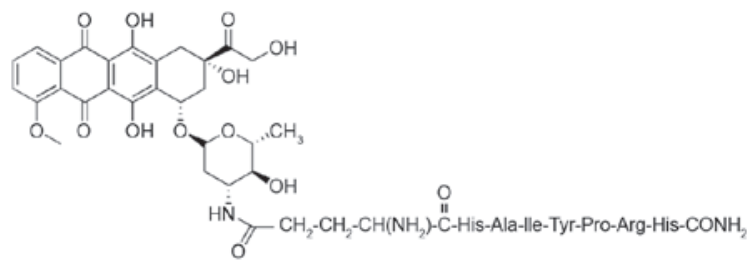

Figure 1. HAIYPRH-EPI conjugate structure. A schematic of the chemical structure of the HAIYPRH-EPI conjugate.

slight modifications. LN229 and U87 cells were washed twice with phosphate-buffered saline (PBS) containing $0.1 \%$ bovine serum albumin and blocked with normal mouse serum at $4^{\circ} \mathrm{C}$ for 60 min prior to staining. A total of $1 \times 10^{6}$ cells were incubated with fluorescein isothiocyanate-conjugated anti-human CD71 (anti-TfR) at $4^{\circ} \mathrm{C}$ for $30 \mathrm{~min}$ and were subsequently washed twice with PBS containing $0.1 \%$ bovine serum albumin. The cells were subsequently analyzed using a flow cytometer (FACSCalibur; BD Biosciences, Franklin Lakes, NJ, USA). An isotype fluorescein isothiocyanate-conjugated IgG1 was used as a negative control to define the threshold of the background staining.

Cytotoxicity evaluation. The cytotoxicity of the conjugate was evaluated in each glioma cell line using a 3-(4,5-dimethylthiazol)-2,5-diphenyltetrazolium (MTT) assay (18). Cells in suspension, containing $\sim 1 \times 10^{4}$ cells, were seeded into each well of a 96-well plate. These cells were treated with the HAIYPRH-EPI conjugate $(0.01-100 \mu \mathrm{M})$ for $24 \mathrm{~h}$ at $37^{\circ} \mathrm{C}$. To test whether the cytotoxicity was enhanced by the addition of free Tf, all cells treated with the HAIYPRH-EPI conjugate were assessed in the presence or absence of free $\operatorname{Tf}(25 \mu \mathrm{M})$. Free EPI and the appropriate culture medium were used as controls. After $24 \mathrm{~h}$, the culture medium was removed, and the cells were incubated with MTT $(5 \mathrm{mg} / \mathrm{ml})$ at $37^{\circ} \mathrm{C}$ in a humidified incubator containing $5 \% \mathrm{CO}_{2}$ for $4 \mathrm{~h}$. Following the removal of the supernatant, the formazan crystals were dissolved in $200 \mu 1$ dimethyl sulfoxide. The absorbance was recorded using a microplate reader (Model 550; Bio-Rad Laboratories, Inc., Hercules, CA, USA) at a wavelength of $570 \mathrm{~nm}$. Cell viability was calculated as $100 \%$ x (absorption treatment $/$ absorption $\left._{\text {control }}\right)$.

Transmission electron microscopy (TEM). LN229 and U87 cells were cultured for $24 \mathrm{~h}$ in 6 -well plates. HAIYPRH-EPI $(10 \mu \mathrm{l})$, EPI $(10 \mu \mathrm{l})$, and the same volume of medium were separately added into treatment and control groups, and the cells were cultured at $37^{\circ} \mathrm{C}$ under a $5 \% \mathrm{CO}_{2}$ atmosphere. After $24 \mathrm{~h}$ of treatment, the cells were harvested, fixed in $2.5 \%$ glutaraldehyde and rinsed with PBS. The cells were subsequently post-fixed in $1 \%$ osmium tetroxide, dehydrated through a series of graded ethanol and acetone, and embedded in epoxy resin. Ultra-thin sections $(60-70 \mathrm{~nm})$ were cut and these sections were stained with $2 \%$ uranyl acetate and lead citrate. Images were captured using a transmission electron microscope (H-600; Hitachi, Ltd., Tokyo, Japan).

Analysis of caspase activity. The activities of caspase-3, caspase- 8 and caspase- 9 in control and drug-treated cells 
A

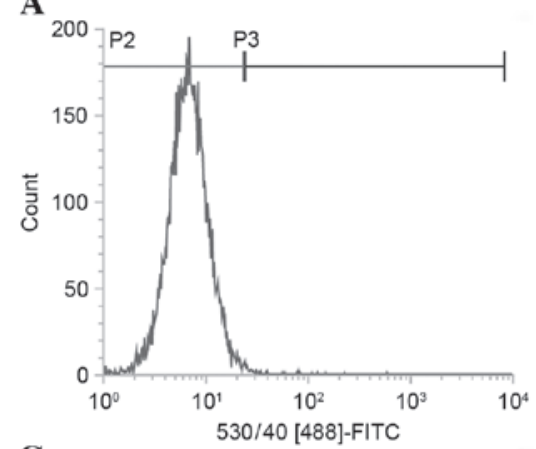

C

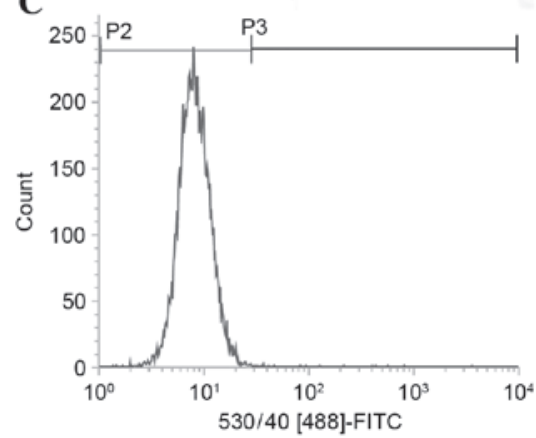

B
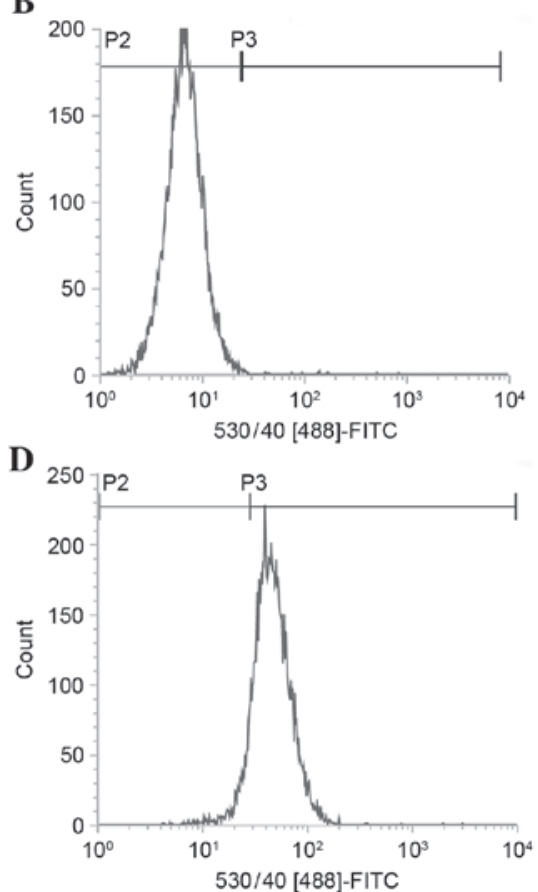

Figure 2. Flow cytometry analyses for TfR surface expressionon LN229 and U87 cells. U87 cells were incubated with (A) isotype FITC-conjugated IgG1 as a negative control or (B) fluorescein isothiocyanate-conjugated anti-human CD71 (anti-TfR). LN229 cells were incubated with (C) isotype FITC-conjugated IgG1 as negative control or (D) fluorescein isothiocyanate-conjugated anti-human CD71 (anti-TfR). TfR, transferrin receptor; FITC, fluorescein isothiocyanate.

were evaluated using caspase activity assay kits (Beyotime Institute of Biotechnology). LN229 and U87 cells were treated separately with HAIYPRH-EPI $(10 \mu \mathrm{l})$, EPI $(10 \mu \mathrm{l})$ or the same volume of medium. Following a $24 \mathrm{~h}$ treatment period, LN229 and U87 cells were lysed using lysis buffer (Beyotime Institute of Biotechnology, Haimen, China). The cell lysates were centrifuged at $26,487 \mathrm{x}$ g for $10 \mathrm{~min}$ at $4^{\circ} \mathrm{C}$. The cell lysate supernatant was mixed with buffer containing Ac-DEVD-pNA (for caspase-3), Ac-IETD-pNA (for caspase-8), and Ac-LEHD-pNA (for caspase-9). The release of pNA was quantified by determining the absorbance using a microplate reader (Model 550; Bio-Rad Laboratories, Inc.) at $405 \mathrm{~nm}$. Caspase activities were expressed as a relative percentage of the control value.

Evaluation of cellular uptake. The cellular uptake of EPI was visualized by fluorescence microscopy. LN229 and U87 cells $\left(1 \times 10^{5}\right.$ cells/well) were seeded separately into 24 -well plates. Following incubation for $24 \mathrm{~h}$, the culture media containing free EPI or HAIYPRH-EPI $(10 \mu \mathrm{M})$ with or without free $\mathrm{Tf}$ $(25 \mu \mathrm{M})$ was added. After a further 2 -h incubation, the treated cells were thoroughly washed three times with PBS to remove the excessive drug that was not taken up by the cells. The fluorescence intensity (FI) of the cells was detected using fluorescence microscopy (Eclipse TE2000-U; Nikon Corporation, Tokyo, Japan).

The extent of cellular uptake was further determined using a flow cytometer (FACSCalibur; BD Biosciences, Franklin Lakes, NJ, USA). LN229 and U87 cells harvested during the logarithmic growth phase were seeded into 24-well plates at a density of $1 \times 10^{6}$ cells/well. Following incubation for $24 \mathrm{~h}$, the culture media containing free EPI or HAIYPRH-EPI
(10 $\mu \mathrm{M})$ was added. Following a further 2-h incubation, the treated cells were thoroughly washed three times with PBS.

Statistical analysis. The results were expressed as the mean \pm standard deviation and were obtained from at least three independent experiments. The statistical analysis of the data was performed using a one-way analysis of variance or Student's t-test. $\mathrm{P}<0.05$ was considered to indicate a statistically significant difference.

\section{Results}

Flow cytometry analyses for TfR surface expression. To identify the difference in the surface expression of TfR between LN229 and U87 cells, flow cytometry was used. The FI reflected the expression of surface TfR. As demonstrated in Fig. 2, the U87 cell line did not express detectable surface TfR and the FI levels of surface TfR did not demonstrate significant differences when compared with their negative control (Fig. 2A). By contrast, in the LN229 cell line surface TfR was detected and the FI levels were higher when compared with the corresponding negative control (Fig. 2B).

Cytotoxicity of HAIYPRH-EPI. The cytotoxicity of the HAIYPRH-EPI conjugate and free EPI was determined to evaluate the in vitro anticancer effects of each condition. As presented in Fig. 3, the HAIYPRH-EPI conjugate exhibited higher cytotoxicity compared with free EPI for the LN229 cell line $(\mathrm{P}<0.05$; Fig. 3A). By contrast, the conjugate exhibited lower cytotoxicity in the U87 cell line compared with free EPI $(\mathrm{P}<0.05$; Fig. 3B). In addition, when Tf was added, the cytotoxicity of HAIYPRH-EPI for the LN229 cells was increased 

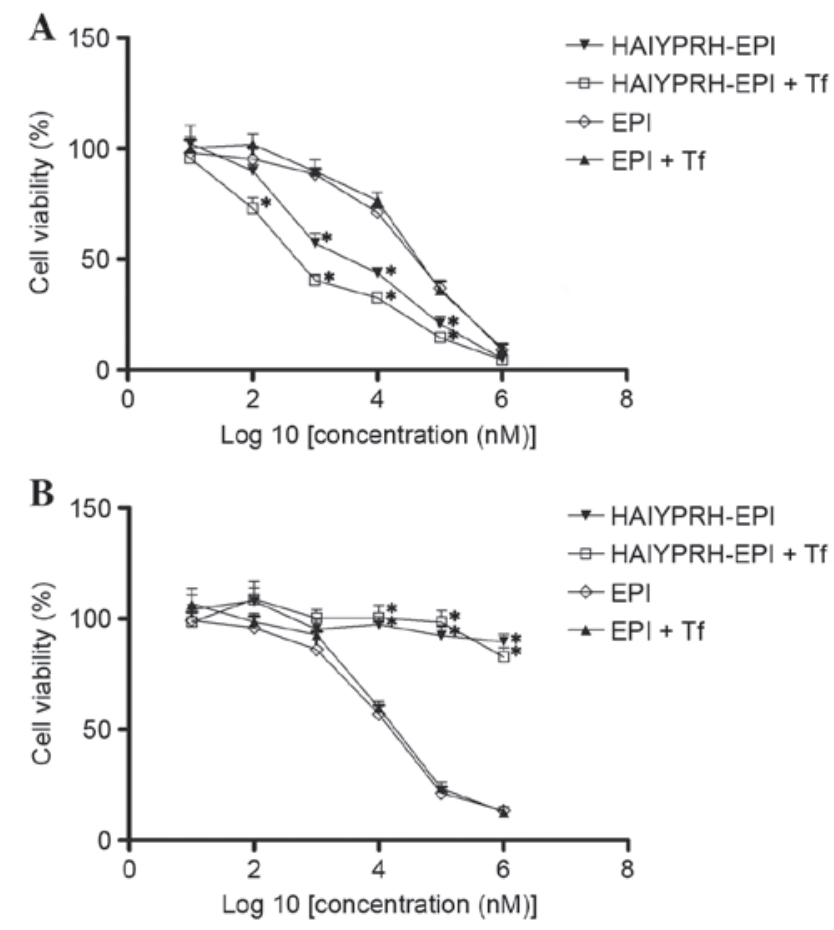

Figure 3. Cytotoxicity of HAIYPRH-EPI and free EPI in LN229 and U87 cells. Cell viability was calculated as (absorption treatment $/$ absorption $_{\text {con- }}$ trol) x 100. (A) LN229 and (B) U87 cells were incubated in 96-well plates and treated with HAIYPRH-EPI or free EPI, with or without free Tf. The curve was fitted using GraphPad Prism version 5.0. All data are presented as the mean \pm standard deviation ( $\mathrm{P}<0.05$ vs. EPI group). EPI, epirubicin; Tf, transferrin.

$(\mathrm{P}<0.05$; Fig. 3A). However, Tf caused no effect on the cytotoxicity of HAIYPRH-EPI for U87 cells ( $\mathrm{P}>0.05$; Fig. $3 \mathrm{~B}$ ) or the cytotoxicity of EPI for the two cell lines.
TEM analysis of cell morphology. TEM is the most common method used for morphological observation by clearly differentiating cellular ultrastructure. In the present study, changes in the nuclei and organelles were observed using TEM. As demonstrated in Fig. 4, untreated U87 cells exhibited intact cell membranes with dense cellular contents. Numerous organelles, including the mitochondria and rough endoplasmic reticulum, were easily observed. The nuclei were large and the nuclear membrane was unbroken. Compared with the control, treatment of the U87 cells with HAIYPRH-EPI $(10 \mu \mathrm{M})$, induced mild apoptosis, and a few myelin figures and fatty drops (white arrows) were observed. The U87 cells treated with only EPI $(10 \mu \mathrm{M})$ exhibited severe apoptosis accompanied with clear apoptotic bodies. By contrast, both groups of LN229 cells treated with EPI $(10 \mu \mathrm{M})$ or HAIYPRH-EPI $(10 \mu \mathrm{M})$ exhibited severe apoptosis.

Analysis of caspase activity. Caspases are significant effector molecules involved in the execution of apoptosis (19). When the LN229 cells were treated with EPI or HAIYPRH-EPI for $24 \mathrm{~h}$, the activity of caspase-3 increased in both groups (Fig. 5A, $\mathrm{P}<0.01)$; however, the activities of caspase- 8 or caspase-9 were not affected by EPI or HAIYPRH-EPI (Fig. 5A, P>0.05). When the U87 cells were treated with EPI or HAIYPRH-EPI for $24 \mathrm{~h}$, the activities of caspase-3 increased only in the EPI group (Fig. 5B, $\mathrm{P}<0.01$ ), and the activities of caspase- 8 or caspase-9 remained unaffected by EPI or HAIYPRH-EPI (Fig. 5A, P>0.05).

Evaluation of cellular uptake. Since EPI itself harbors florescence, the uptake of free EPI and HAIYPRH-EPI by the LN229 and U87 cells was investigated using the methods of fluorescence microscopy and flow cytometry. As demonstrated in Fig. 6, when incubated with HAIYPRH-EPI, the
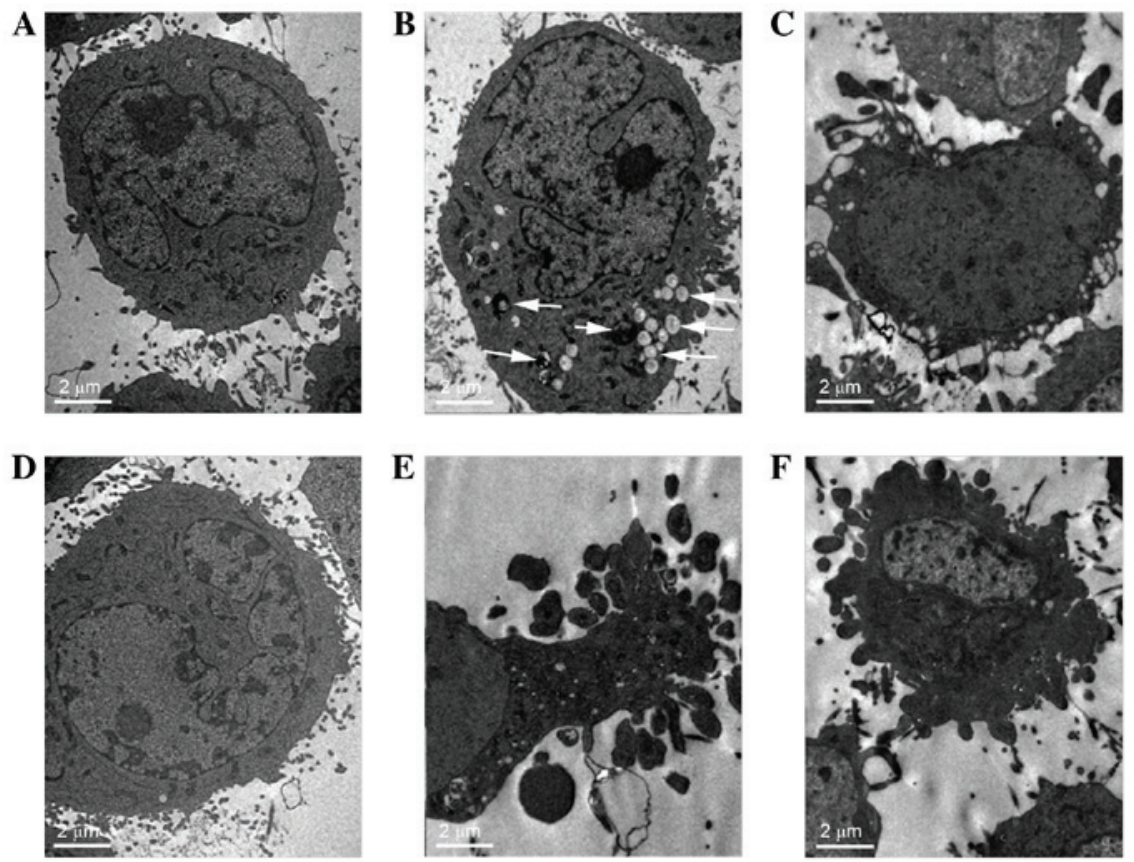

Figure 4. Morphological analysis of the effects of HAIYPRH-EPI or free EPI by TEM. (A) Untreated U87 cells, (B) U87 cells treated with HAIYPRH-EPI $(10 \mu \mathrm{M}),(\mathrm{C}) \mathrm{U} 87$ cells treated with EPI $(10 \mu \mathrm{M})$, (D) untreated LN229 cells, (E) LN229 cells treated with HAIYPRH-EPI (10 $\mu \mathrm{M})$ and (F) LN229 cells treated with EPI $(10 \mu \mathrm{M})$ were assessed by TEM. EPI, epirubicin; TEM, transmission electron microscopy. 
A

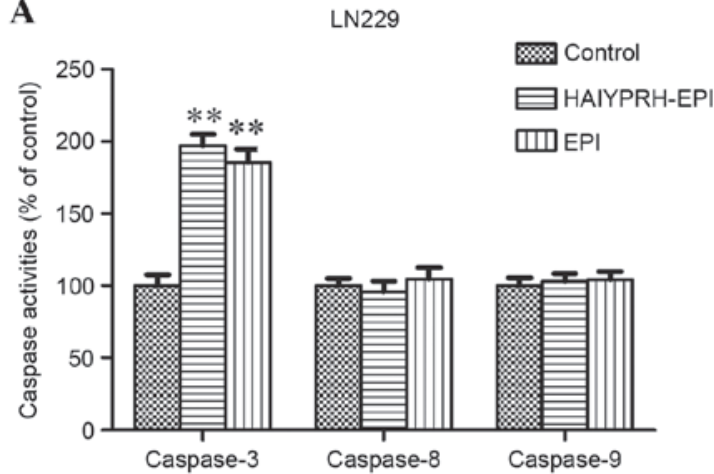

B

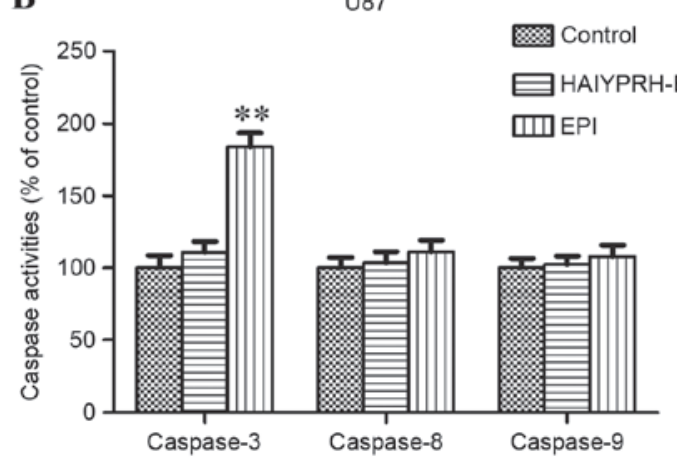

Figure 5. Analysis of caspase activity. The activity of caspase-3, -8 and -9 was assessed in (A) LN229 and (B) U87 cells using relevant caspase activity assay kits. All data are presented as the mean \pm standard deviation $\left({ }^{*} \mathrm{P}<0.05\right.$ and ${ }^{* * *} \mathrm{P}<0.01$ vs. Control). EPI, epirubicin.

LN229 cells emitted a higher fluorescence intensity compared with the U87 cells, suggesting that the HAIYPRH-EPI cellular uptake of LN229 cells was higher compared with that of U87 cells. Furthermore, the addition of Tf significantly increased the intracellular uptake of HAIYPRH-EPI in LN229 cells. By contrast, when incubated with free EPI, no obvious difference was observed between the fluorescence of LN229 and U87 cells, and the addition of Tf caused no significant change in cellular uptake of EPI inLN229 or U87 cells, when compared with EPI alone. As demonstrated in Fig. 7, similar results were observed by flow cytometry. Flow cytometry also confirmed that the LN229 cells exhibited more uptake of HAIYPRH-EPI compared with the U87 cells, and that LN299 exhibited enhanced uptake of HAIYPRH-EPI with the addition of Tf. The mean fluorescence intensity highlighted these findings (Fig. 8).

\section{Discussion}

Although it has been previously demonstrated that TfR is overexpressed in numerous solid tumor types, the level of TfR varies among different tumor cell lines $(11,20,21)$. Wirth et al (21) detected TfR expression in 41 human tumor cell lines using microarrays. This previous study identified that TfR expression in these cell lines was completely different compared with one another. Furthermore, for glioma cell lines, TfR expression in LN229 cells was positive, but negative in U87 cells. Notably, another study reported that both the LN229 and U87 cell lines overexpressed TfR, as determined by immunofluorescence and western blot
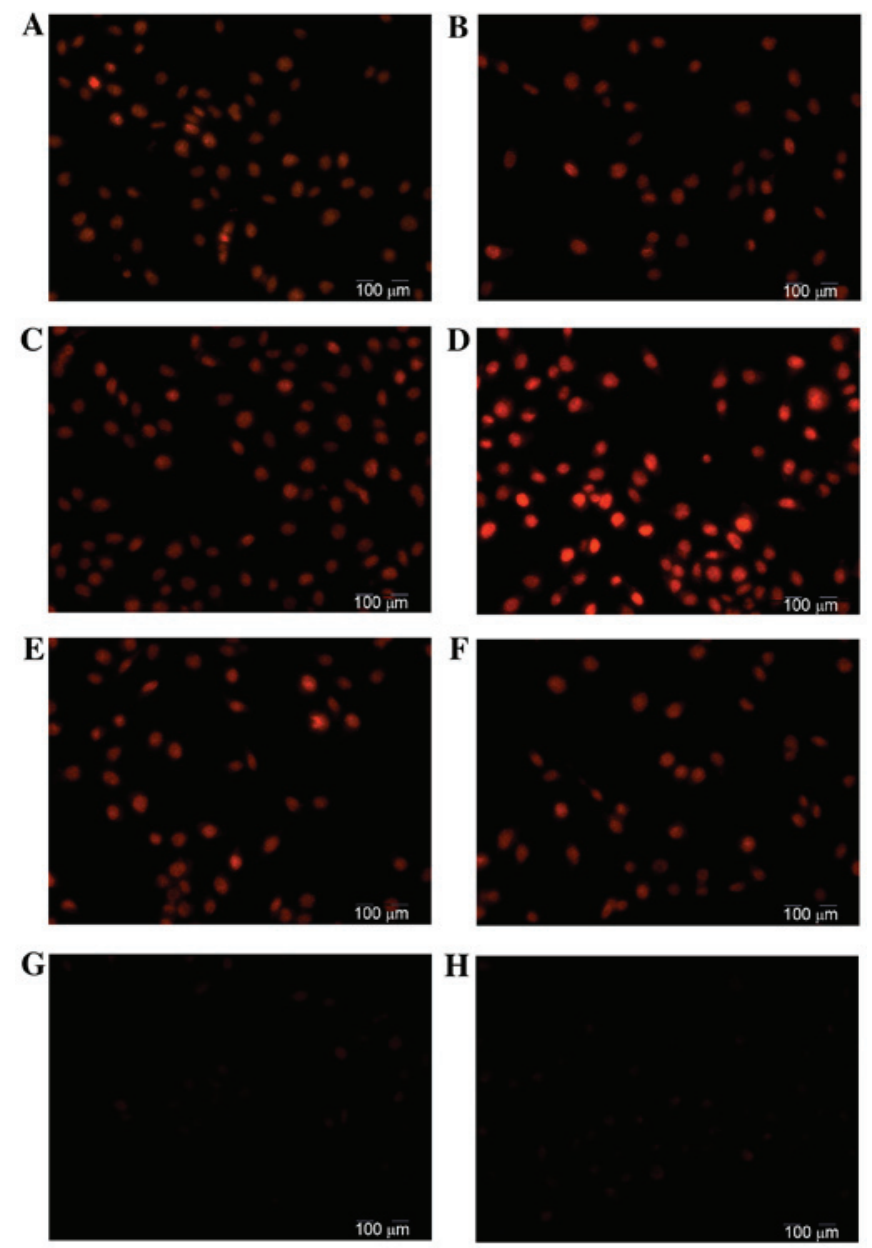

Figure 6. Assessment of HAIYPRH uptake. Since EPI itself harbors florescence, the uptake of free EPI and HAIYPRH-EPI by the LN229 and U87 cells was investigated using immunofluorescence imaging. LN229 cells treated with free (A) EPI $(10 \mu \mathrm{M})$, (B) free EPI $(10 \mu \mathrm{M})$ and Tf $(25 \mu \mathrm{M})$, (C) HAIYPRH-EPI $(10 \mu \mathrm{M})$ or (D) HAIYPRH-EPI $(10 \mu \mathrm{M})$ and Tf $(25 \mu \mathrm{M})$ were assessed by fluorescence microscopy. U87 cells treated with (E) free EPI $(10 \mu \mathrm{M}),(\mathrm{F})$ free EPI $(10 \mu \mathrm{M})$ and Tf $(25 \mu \mathrm{M}),(\mathrm{G})$ HAIYPRH-EPI $(10 \mu \mathrm{M})$ or $(\mathrm{H})$ HAIYPRH-EPI $(10 \mu \mathrm{M})$ and $\mathrm{Tf}(25 \mu \mathrm{M})$ were assessed by fluorescence microscopy. EPI, epirubicin; Tf, transferrin.

analysis (19). Total TfR in cells includes surface TfR and endocellular TfR. The previous study reported that U87 cell lines overexpressed TfR, which was the total TfR, not surface TfR (19). Considering that surface TfR, not endocellular TfR, executes the delivery of $\mathrm{Tf}$ or drugs across cell membrane and the controversy surrounding the expression of TfR in LN229 and U87 cells (19,21), the difference in surface TfR expression between LN229 and U87 cells was examined in the present study. The results demonstrated that surface TfR expression in LN229 cells was positive, but the U87 levels had no difference compared with the negative control (Fig. 2), which was similar to the results reported by Wirth et al (21).

The results of the cytotoxicity assay indicated that the cytotoxicity of HAIYPRH-EPI was correlated with the expression of surface TfR. Surface TfR was expressed at greater levels on the surface of LN229 cells when compared with U87 cells, and due to the high affinity of HAIYPRH, it mediated the endocytosis of HAIYPRH-EPI. On the contrary, surface TfR was expressed at lower levels in U87 cells; thus, the active transport of HAIYPRH-EPI by surface TfR was rare in these 

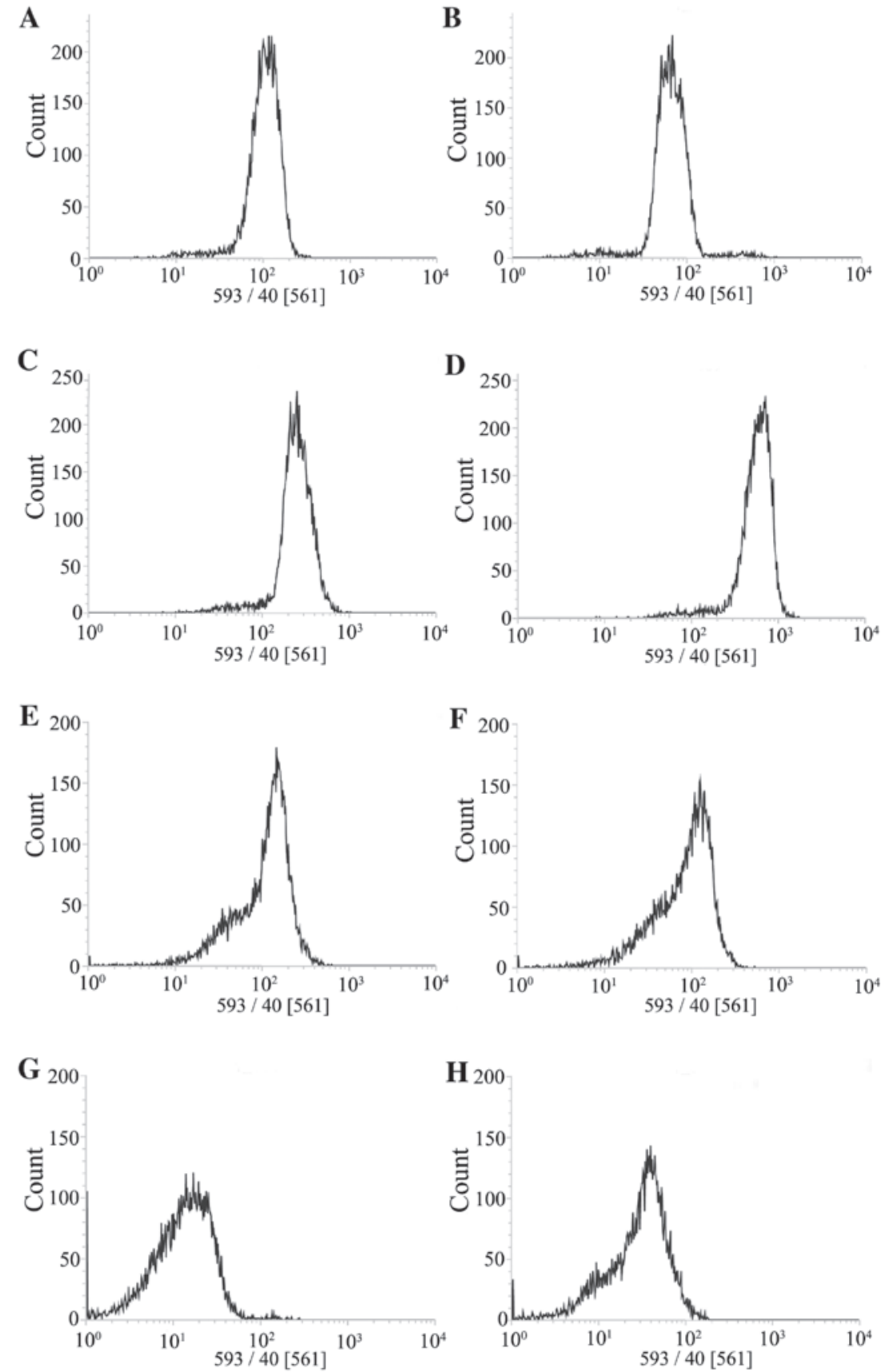

Figure 7. Flow cytometry analysis of cellular uptake of HAIYPRH in LN229 and U87 cells. Since EPI itself harbors florescence, the uptake of free EPI and HAIYPRH-EPI by the LN229 and U87 cells was investigated using flow cytometry. LN229 cells treated with (A) free EPI (10 $\mu \mathrm{M})$, (B) free EPI (10 $\mu \mathrm{M})$ and $\operatorname{Tf}(25 \mu \mathrm{M})$, (C) HAIYPRH-EPI $(10 \mu \mathrm{M})$ or (D) HAIYPRH-EPI $(10 \mu \mathrm{M})$ and Tf $(25 \mu \mathrm{M})$ were assessed by flow cytometry. U87 cells treated with (E) free EPI $(10 \mu \mathrm{M}),(\mathrm{F})$ free EPI $(10 \mu \mathrm{M})$ and Tf $(25 \mu \mathrm{M}),(\mathrm{G})$ HAIYPRH-EPI $(10 \mu \mathrm{M})$ or $(\mathrm{H})$ HAIYPRH-EPI $(10 \mu \mathrm{M})$ and Tf $(25 \mu \mathrm{M})$ were assessed by flow cytometry. EPI, epirubicin; Tf, transferrin.

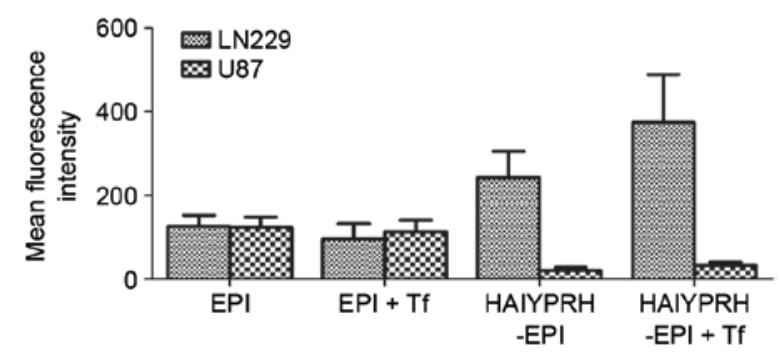

Figure 8. Mean fluorescence intensity of LN229 and U87 cells determined by flow cytometry. LN229 and U87 cells were treated with free EPI $(10 \mu \mathrm{M})$, free $\mathrm{EPI}(10 \mu \mathrm{M})$ and $\mathrm{Tf}(25 \mu \mathrm{M})$, HAIYPRH-EPI $(10 \mu \mathrm{M})$ or HAIYPRH-EPI $(10 \mu \mathrm{M})$ and $\operatorname{Tf}(25 \mu \mathrm{M})$ prior to assessment by flow cytometry. cells. By contrast, the cellular uptake of HAIYPRH-EPI through passive transport was more difficult to achieve compared with the transport of free EPI, due to the strong hydrophilicity of HAIYPRH. Therefore, HAIYPRH-EPI exhibited lower cytotoxicity in U87 cells and higher toxicity in LN229 cells.

To corroborate the cytotoxicity results, TEM was used to determine cell apoptosis through morphological observation of the cellular ultrastructure, and similar results were observed. When cells are exposed to apoptotic stimuli, there are two major apoptosis signaling pathways: Intrinsic (mitochondria-mediated) pathway and extrinsic (transmembrane 
receptor-mediated) pathway, both of which use caspases, a family of cysteine proteases (22). Caspases are divided into two groups: Initiator caspases and effector caspases. When initiator caspases bind to adaptor molecules, they are activated, and are able to activate effector caspases. The major initiator caspase for the intrinsic pathway is caspase-9, whereas the major initiator caspase for extrinsic pathway is caspase-8. Each pathway shares the same effector caspase, caspase-3, which is a key checkpoint enzyme in the canonical apoptotic pathway $(22,23)$. To elucidate the initiation event of apoptosis, the present study probed the activation of the two initiator caspases, caspase- 8 and caspase- 9 , and the effector caspase, caspase-3. The results demonstrated that, when treated with EPI or HAIYPRH-EPI, the activity of caspase-3 increased, whilst the activities of caspase- 8 and caspase- 9 did not. A previous study also reported that EPI $(0.5 \mu \mathrm{g} / \mathrm{ml})$ induced the activation of caspase-3, but not caspase- 8 or caspase-9, in $\mathrm{HeLa}$ cells (24). These results suggested that the pro-apoptotic effect of EPI or HAIYPRH-EPI was not mediated by caspase- 8 or caspase- 9 , and that caspase- 3 may be activated by additional upstream factors.

Consistent with the cytotoxicity and cell apoptosis data, the results of the cellular uptake experiments further confirmed the original hypothesis. HAIYPRH-EPI is a hydrophilic substance, and its transport across the cell membrane predominantly depends on active transport mediated by surface TfR, as opposed to passive transport. The different expression levels of surface TfR on LN229 and U87 cells led to different cellular uptakes of HAIYPRH-EPI, further resulting in different levels of cytotoxicity. Due to the higher expression of surface TfR, more HAIYPRH-EPI can be transported into LN229 cells. Therefore, HAIYPRH-EPI was markedly more active in LN229 cells compared with in U87 cells. By contrast, EPI is a relatively hydrophobic substance. Its main transport was passive, so the cellular uptake of free EPI was not different between LN229 and U87 cells in the present study.

The primary function of $\mathrm{Tf}$ is serum iron transportation and it can reversibly bind two atoms of ferric iron with high affinity (11). Tf exhibits a conformational change following iron binding, which has been demonstrated to be significant in its selective recognition by the TfR (25). Following binding to the TfR, the Tf-TfR complex activates a cascade that is suggested to be important in the mediation of its specific internalization (26). Therefore, the internalization mediated by TfR is triggered by $\mathrm{Tf}$ (12). As mentioned previously, the binding sites of $\mathrm{Tf}$ and $\mathrm{TfR}$ are distinct. Therefore, the addition of $\mathrm{Tf}$ can theoretically enhance the uptake of HAIYPRH-EPI in LN229 cells, and the results examining the cytotoxicity and cellular uptake also confirmed this hypothesis.

In conclusion, the cytotoxicity of HAIYPRH-EPI was dependent upon the expression of the surface transferrin receptor. Considering that the majority of cancer cells have high rates of iron uptake, and that TfR is generally overexpressed on cancer cells, the authors speculated that the HAIYPRH-EPI conjugate may provide an effective strategy for increasing the selectivity of EPI for cancer cells and reduce the systemic toxicity of EPI. To confirm the hypothesis, the therapeutic effects and side effects of HAIYPRH-EPI with a suitable delivery system in an animal model will be investigated in a future study.

\section{Acknowledgements}

The present study was supported by the National Science and Technology Major Project (no. 2010ZX09401-306-1-1).

\section{References}

1. Hu DG, Rogers A and Mackenzie PI: Epirubicin upregulates UDP glucuronosyltransferase 2B7 expression in liver cancer cells via the p53 pathway. Mol Pharmacol 85: 887-897, 2014.

2. Khasraw M, Bell R and Dang C: Epirubicin: Is it like doxorubicin in breast cancer? A clinical review. Breast 21: 142-149, 2012.

3. Miyoshi Y, Kurosumi M, Kurebayashi J, Matsuura N, Takahashi M, Tokunaga E, Egawa C, Masuda N, Kim SJ, Okishiro M, et al: Topoisomerase IIalpha-positive and BRCA1-negative phenotype: Association with favorable response to epirubicin-based regimens for human breast cancers. Cancer Lett 264: 44-53, 2008.

4. Okishiro M, Kim SJ, Tsunashima R, Nakayama T, Shimazu K, Shimomura A, Maruyama N, Tamaki Y and Noguchi S: MDM2 SNP309 and TP53 R72P associated with severe and febrile neutropenia in breast cancer patients treated with 5-FU/epirubicin/cyclophosphamide. Breast Cancer Res Treat 132: 947-953, 2012.

5. Aogi K, Saeki T, Nakamura S, Kashiwaba M, Sato N, Masuda N, Rai Y, Ohno S, Kuroi K, Nishimura R, et al: A multicenter, phase II study of epirubicin/cyclophosphamide followed by docetaxel and concurrent trastuzumab as primary systemic therapy for HER-2 positive advanced breast cancer (the HER2NAT study). Int J Clin Oncol 18: 598-606, 2013.

6. Nasr M, Nafee N, Saad H and Kazem A: Improved antitumor activity and reduced cardiotoxicity of epirubicin using hepatocyte-targeted nanoparticles combined with tocotrienols against hepatocellular carcinoma in mice. Eur J Pharm Biopharm 88: 216-225, 2014

7. Appel JM, Zerahn B, Moller S, Christensen HM, Søgaard P, Ejlertsen B, Fogh-Andersen N, Jensen BV and Nielsen DL: Long-term heart function after adjuvant epirubicin chemotherapy for breast cancer. Acta Oncol 51: 1054-1061, 2012.

8. Ju RJ, Li XT, Shi JF, Li XY, Sun MG, Zeng F, Zhou J, Liu L, Zhang CX, Zhao WY and Lu WL: Liposomes, modified with PTD (HIV-1) peptide, containing epirubicin and celecoxib, to target vasculogenic mimicry channels in invasive breast cancer. Biomaterials 35: 7610-7621, 2014.

9. Haisma HJ, Boven E, van Muijen M, de Jong J, van der Vijgh WJ and Pinedo HM: A monoclonal antibody-beta-glucuronidase conjugate as activator of the prodrug epirubicin-glucuronide for specific treatment of cancer. Br J Cancer 66: 474-478, 1992.

10. Xu J, Sheng Y, Xu F, Yu Y and Chen Y: Quantitative subcellular study of transferrin receptor-targeted doxorubicin and its metabolite in human breast cancer cells. Eur J Drug Metab Pharmacokinet 39: 301-310, 2014.

11. Tortorella S and Karagiannis TC: Transferrin receptor-mediated endocytosis: A useful target for cancer therapy. J Membr Biol 247: 291-307, 2014.

12. Oh S, Kim BJ, Singh NP, Lai H and Sasaki T: Synthesis and anti-cancer activity of covalent conjugates of artemisinin and a transferrin-receptor targeting peptide. Cancer Lett 274: 33-39, 2009.

13. Han L, Huang R, Liu S, Huang S and Jiang C: Peptide-conjugated PAMAM for targeted doxorubicin delivery to transferrin receptor overexpressed tumors. Mol Pharm 7: 2156-2165, 2010.

14. Malarvizhi GL, Retnakumari AP, Nair S and Koyakutty M: Transferrin targeted core-shell nanomedicine for combinatorial delivery of doxorubicin and sorafenib against hepatocellular carcinoma. Nanomedicine 10: 1649-1659, 2014.

15. Nam JP, Park SC, Kim TH, Jang JY, Choi C, Jang MK and Nah JW: Encapsulation of paclitaxel into lauric acid-O-carboxymethyl chitosan-transferrin micelles for hydrophobic drug delivery and site-specific targeted delivery. Int J Pharm 457: 124-135, 2013.

16. Lee JH, Engler JA, Collawn JF and Moore BA: Receptor mediated uptake of peptides that bind the human transferrin receptor. Eur J Biochem 268: 2004-2012, 2001.

17. Kato J, Kobune M, Ohkubo S, Fujikawa K, Tanaka M, Takimoto R, Takada K, Takahari D, Kawano Y, Kohgo Y and Niitsu Y: Iron/IRP-1-dependent regulation of mRNA expression for transferrin receptor, DMT1 and ferritin during human erythroid differentiation. Exp Hematol 35: 879-887, 2007. 
18. Qian G, Wang Z, Zhao J, Li D, Gao W, Wang B, Sui D, Qu X and Chen Y: Synthesis and anti-cancer cell activity of pseudo-ginsenoside Rh2. Steroids 92: 1-6, 2014

19. Dixit S, Novak T, Miller K, Zhu Y, Kenney ME and Broome AM: Transferrin receptor-targeted theranostic gold nanoparticles for photosensitizer delivery in brain tumors. Nanoscale 7: 1782-1790, 2015.

20. Xu J, Sheng Y, Xu F, Yu Y and Chen Y: Quantitative subcellular study of transferrin receptor-targeted doxorubicin and its metabolite in human breast cancer cells. Eur J Drug Metab Pharmacokinet 39: 301-310, 2014.

21. Wirth GJ, Schandelmaier K, Smith V, Burger AM and Fiebig HH: Microarrays of 41 human tumor cell lines for the characterization of new molecular targets: Expression patterns of cathepsin B and the transferrin receptor. Oncology 71: 86-94, 2006.

22. Lo YL, Ho CT and Tsai FL: Inhibit multidrug resistance and induce apoptosis by using glycocholic acid and epirubicin. Eur J Pharm Sci 35: 52-67, 2008
23. Pakunlu RI, Wang Y, Saad M, Khandare JJ, Starovoytov V and Minko T: In vitro and in vivo intracellular liposomal delivery of antisense oligonucleotides and anticancer drug. J Control Release 114: 153-162, 2006.

24. Lin Y, Jiang D, Li Y, Han X, Yu D, Park JH and Jin YH: Effect of sun ginseng potentiation on epirubicin and paclitaxel-induced apoptosis in human cervical cancer cells. J Ginseng Res 39: 22-28, 2015.

25. Richardson DR and Ponka P: The molecular mechanisms of the metabolism and transport of iron in normal and neoplastic cells. Biochim Biophys Acta 1331: 1-40, 1997.

26. Yashunsky V, Shimron S, Lirtsman V, Weiss AM, Melamed-Book N, Golosovsky M, Davidov D and Aroeti B: Real-time monitoring of transferrin-induced endocytic vesicle formation by mid-infrared surface plasmon resonance. Biophys J 97: 1003-1012, 2009. 